\title{
Mass spectra of mesons containing charm quarks - continuum limit results from twisted mass fermions
}

\section{Krzysztof Cichy*}

Goethe-Universität Frankfurt am Main, Institut für Theoretische Physik, Max-von-Laue-Str. 1, D-60438 Frankfurt am Main, Germany

Adam Mickiewicz University, Faculty of Physics, Umultowska 85, 61-614 Poznań, Poland

E-mail: kcichyath.physik-uni-frankfurt.de

\section{Martin Kalinowski}

Goethe-Universität Frankfurt am Main, Institut für Theoretische Physik, Max-von-Laue-Str. 1, D-60438 Frankfurt am Main, Germany

E-mail: kalinowmeth.physik-uni-frankfurt.de

\section{Marc Wagner}

Goethe-Universität Frankfurt am Main, Institut für Theoretische Physik, Max-von-Laue-Str. 1, D-60438 Frankfurt am Main, Germany

E-mail: mwagnereth.physik-uni-frankfurt.de

\begin{abstract}
We present results from an ongoing computation of masses of $D$ mesons, $D_{s}$ mesons and charmonium, including both ground states and several parity and angular momentum excitations. We employ 2+1+1 flavours of dynamical maximally twisted mass fermions at three lattice spacings and three $u / d$ quark masses at each lattice spacing. We consider different combinations of valence quark discretizations, with either identical or opposite signs in front of the twisted mass terms. In the end, our setup allows for a good control of different kinds of systematic effects, in particular the quark mass dependence of the resulting meson masses and cut-off effects. We obtain good agreement with experiment for the majority of states and we discuss improvements that will be made to finalize the analysis.
\end{abstract}

The 33rd International Symposium on Lattice Field Theory

14 -18 July 2015

Kobe International Conference Center, Kobe, Japan

* Speaker. 


\section{Introduction}

Meanwhile, there is a large number of mesons known experimentally which contain charm quarks. Some of them are well established and in good agreement with phenomenological expectations, but in other cases their masses and/or widths are not well understood theoretically. For example, the $D_{s 0}^{*}$ and $D_{s 1}$ mesons are conjectured to be tetraquark candidates or mixtures of a mesonic and a tetraquark structure. Hence, an ab initio investigation of charmed mesons is highly interesting and can be in principle realized on the lattice. However, charm physics on the lattice is complicated due to the currently feasible values of the lattice spacing - if they are too coarse, the charm quark mass is large in lattice units. Nevertheless, with current computational resources many questions can be addressed, including the spectrum of charmed mesons. Moreover, charm quarks can be treated as dynamical, so all systematic effects can be controlled with reasonable precision. For recent lattice QCD papers using quark-antiquark operators for charm quark containing mesons, see e.g. Refs. [1, 2, 3, 4, 5, 6, 7, 8, 9, 10, 11, 12, 13]. For papers using additionally fourquark interpolating operators, cf. Refs. $[14,15,16,17,18,19,20,21]$. Our goal is to compute the spectrum of $D$ mesons (charm-light), $D_{s}$ mesons (charm-strange) and charmonium (charm-charm) using fully dynamical twisted mass ensembles generated by the European Twisted Mass Collaboration (ETMC) with 2+1+1 flavours. The results reported in this proceeding are from an ongoing work aiming at extending Ref. [22] to obtain the results in the continuum limit.

\section{Lattice setup and lattice techniques}

We use dynamical twisted mass (TM) configurations generated by ETMC with $2+1+1$ dynamical quark flavours [23]. The gauge action is the Iwasaki action [24], while the fermionic sector consists of the Wilson twisted mass action for the degenerate up/down doublet [25] and nondegenerate strange/charm doublet [26]. Automatic $\mathscr{O}(a)$ improvement is realized by setting the hopping parameter $\kappa$ to its critical value for which the PCAC quark mass vanishes [25, 27].

In the valence sector, we use the following setup. The action for the light quarks is the same as the one in the sea. For strange and charm, we introduce two strange $\left(s, s^{\prime}\right)$ and two charm $\left(c, c^{\prime}\right)$ quark flavours with the action for a single flavour $f$ [27]:

$$
D_{f}=D_{W}+m_{0}+i \mu_{f} \gamma_{5} .
$$

We take

- either $\mu_{s / c}=-\mu_{s^{\prime} / c^{\prime}}$ - we call this TM setup (however, it is still non-unitary)

- or $\mu_{s / c}=\mu_{s^{\prime} / c^{\prime}}$ - we call this Osterwalder-Seiler (OS) setup.

In this way, we avoid the mixing of strange and charm quarks, which would make the computations problematic. It is important to emphasize that such setup still guarantees automatic $\mathscr{O}(a)$ improvement.

The simulation parameters of our ensembles are summarized in Tab. 1. We use three lattice spacings between approximately $0.06 \mathrm{fm}$ and $0.09 \mathrm{fm}$ and pion masses ranging between around $230 \mathrm{MeV}$ and $480 \mathrm{MeV}$. This enables us to investigate the discretization and quark mass effects and extrapolate our results to the continuum limit and the physical pion mass. 


\begin{tabular}{ccccccccc} 
Ensemble & $\beta$ & lattice & $a \mu_{l}$ & $\begin{array}{c}\mu_{l, R} \\
{[\mathrm{MeV}]}\end{array}$ & $\kappa_{c}$ & $\begin{array}{c}L \\
{[\mathrm{fm}]}\end{array}$ & $m_{\pi} L$ & $\begin{array}{c}a \\
{[\mathrm{fm}]}\end{array}$ \\
\hline $\mathrm{A} 30.32$ & 1.90 & $32^{3} \times 64$ & 0.0030 & 13 & 0.163272 & 2.8 & 3.5 & 0.0885 \\
$\mathrm{~A} 40.32$ & 1.90 & $32^{3} \times 64$ & 0.0040 & 17 & 0.163270 & 2.8 & 4.1 & 0.0885 \\
$\mathrm{~A} 80.24$ & 1.90 & $24^{3} \times 48$ & 0.0080 & 34 & 0.163260 & 2.1 & 4.3 & 0.0885 \\
$\mathrm{~B} 25.32$ & 1.95 & $32^{3} \times 64$ & 0.0025 & 12 & 0.161240 & 2.6 & 3.2 & 0.0815 \\
$\mathrm{~B} 55.32$ & 1.95 & $32^{3} \times 64$ & 0.0055 & 26 & 0.161236 & 2.6 & 4.6 & 0.0815 \\
$\mathrm{D} 15.48$ & 2.10 & $48^{3} \times 96$ & 0.0015 & 9 & 0.156361 & 3.0 & 3.2 & 0.0619 \\
$\mathrm{D} 20.48$ & 2.10 & $48^{3} \times 96$ & 0.0020 & 12 & 0.156357 & 3.0 & 3.7 & 0.0619 \\
$\mathrm{D} 30.48$ & 2.10 & $48^{3} \times 96$ & 0.0030 & 19 & 0.156355 & 3.0 & 4.5 & 0.0619
\end{tabular}

Table 1: Simulation parameters for gauge field configuration ensembles used in this work. Shown are: ensemble label, inverse gauge coupling $(\beta)$, lattice volume $\left((L / a)^{3} \times(T / a)\right)$, sea quark mass $\left(a \mu_{l}\right)$, its physical value in $\mathrm{MeV}\left(\mu_{l, R}\right.$, renormalized in the $\overline{\mathrm{MS}}$ scheme at $\left.\mu=2 \mathrm{GeV}\right)$, critical value of the hopping parameter yielding vanishing PCAC mass $\left(\kappa_{c}\right)$, lattice extent in $\mathrm{fm}(L)$, product of the pion mass and the lattice extent $\left(m_{\pi} L\right)$ and lattice spacing in $\mathrm{fm}(a)$.

Our lattice meson creation operators are of the following form ${ }^{1}$ :

$$
O_{\Gamma, \bar{\chi}^{(1)} \chi^{(2)}}^{\mathrm{tm}} \equiv \frac{1}{\sqrt{V / a^{3}}} \sum_{\mathbf{n}} \bar{\chi}^{(1)} \underset{\Delta \mathbf{n}= \pm \mathbf{e}_{x}, \pm \mathbf{e}_{y}, \pm \mathbf{e}_{z}}{(\mathbf{n})} \sum_{i} U(\mathbf{n}+\mathbf{n}) \Gamma(\Delta \mathbf{n}) \chi^{(2)}(\mathbf{n}+\Delta \mathbf{n})
$$

where $\sum_{\mathbf{n}}$ gives zero total momentum, $\sum_{\Delta \mathbf{n}}$ realizes spatial separation between quarks (such that the meson can have orbital angular momentum), $\Gamma(\Delta \mathbf{n})$ is a suitable combination of spherical harmonics and $\gamma$-matrices (determines total angular momentum, parity and charge conjugation properties (for charmonium) $), U(\mathbf{n} ; \mathbf{n}+\Delta \mathbf{n})$ is a gauge link, $\chi^{(1),(2)}$ are twisted basis quark operators.

We use standard smearing techniques to enhance the overlap between trial states and low lying meson states. We apply APE smearing of links and Gaussian smearing of quark fields. Note that smearing does not affect the irreducible representation of the cubic group, equivalent of the total angular momentum $\mathrm{O}^{J}$ on the lattice, parity $\mathscr{P}$ and charge conjugation $\mathscr{C}$, all determined by $\Gamma(\Delta \mathbf{n})$ in the creation operators.

For each sector, i.e. the same flavours $\bar{\chi}^{(1)} \chi^{(2)}$, cubic representation $\mathrm{O}^{J}$ and (for $\left.\bar{c} c\right) \mathscr{C}(\mathrm{OS})$ or $\mathscr{C} \circ \mathscr{P}^{(\mathrm{tm})}(\mathrm{TM})$, we compute temporal correlation matrices of meson creation operators. The different entries in a given correlation matrix hence differ by their $\Gamma$-structure (spin) and parity $\mathscr{P}$, since parity is broken by TM at finite lattice spacing. An example of extraction of meson masses and assignment of parity from a $2 \times 2$ correlation matrix is discussed in detail in Ref. [22], Sec. 4.2.

\section{Results}

\subsection{Extrapolation procedure}

Our aim is to extract the physical masses of mesons containing charm quarks. Therefore, we use the following procedure to extrapolate to the continuum limit and interpolate/extrapolate to the physical quark masses:

\footnotetext{
${ }^{1}$ For a detailed account of the used lattice techniques, we refer to Ref. [22].
} 

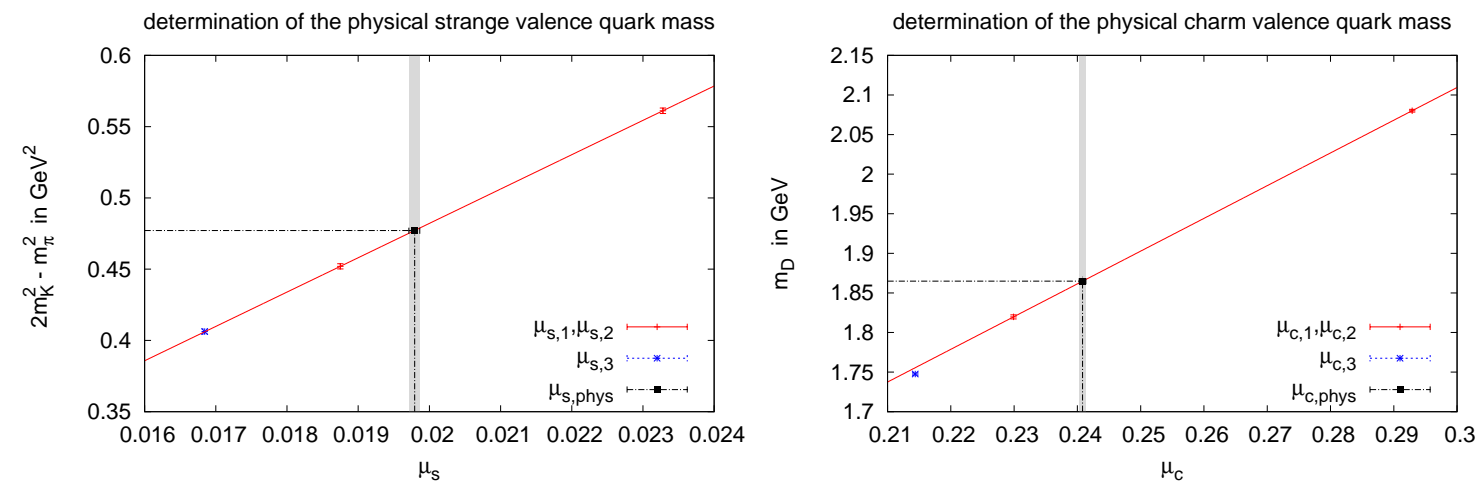

Figure 1: Examples of tuning to physical strange (left) and charm (right) quark masses. Ensemble A80.24.

1. We compute the relevant TM/OS correlation functions for three lattice spacings, three light quark masses for each lattice spacing (with the exception of B-ensembles where the computations for one mass are still in progress), two strange quark masses per light quark mass and two charm quark masses per light quark mass (i.e. three pairs $\left(\mu_{s, 1}, \mu_{c, 2}\right),\left(\mu_{s, 2}, \mu_{c, 1}\right)$, $\left(\mu_{s, 2}, \mu_{c, 2}\right)$ for each light quark mass $\left.\mu_{l}\right)$.

2. We tune the strange/charm quark masses via $2 m_{K}^{2}-m_{\pi}^{2}$ (which does not depend on the light quark mass at leading order of chiral perturbation theory) and the $D$ meson mass, $m_{D}$ (essentially light quark mass independent). To compute $2 m_{K}^{2}-m_{\pi}^{2}$ and $m_{D}$ for this tuning, we always use the TM setup $\left(\mu_{s, c}=-\mu_{s^{\prime}, c^{\prime}}\right)$. The physical strange/charm quark masses are such values of $\mu_{s} / \mu_{c}$ that $2 m_{K}^{2}-m_{\pi}^{2}$ and $m_{D}$ take their physical values of $0.477 \mathrm{GeV}^{2}$ and 1.865 $\mathrm{GeV}$, respectively (cf. Fig. 1).

3. Using the values of the physical strange/charm quark masses $\mu_{s} / \mu_{c}$, we inter-/extrapolate all our meson masses. We use jackknife with binning to account for autocorrelations and propagate the errors from the tuning.

4. This gives us a set of 16 points per meson mass (3 lattice spacings $\times(2-3)$ light quark masses $\times 2$ discretizations). Having this set of data points, we perform a combined chiral and continuum extrapolation, using the following fitting ansätze:

$$
\begin{gathered}
M^{T M}\left(a, m_{\pi}\right)=M+c^{T M} a^{2}+\alpha^{T M}\left(m_{\pi}^{2}-m_{\pi, p h y s}^{2}\right), \\
M^{O S}\left(a, m_{\pi}\right)=M+c^{O S} a^{2}+\alpha^{O S}\left(m_{\pi}^{2}-m_{\pi, p h y s}^{2}\right)
\end{gathered}
$$

with five fitting parameters: $M, c^{T M}, c^{O S}, \alpha^{T M}, \alpha^{O S}$. Note that we enforce a common continuum and physical pion mass limit $M$ for both discretizations.

\subsection{Examples of combined chiral and continuum extrapolations and spectrum plots}

In Figs. 2 and 3, we present examples of our combined chiral and continuum extrapolations using Eqs. (3.1) and (3.2), four in the charmonium sector and two in the $D_{s}$ sector. In all cases, 

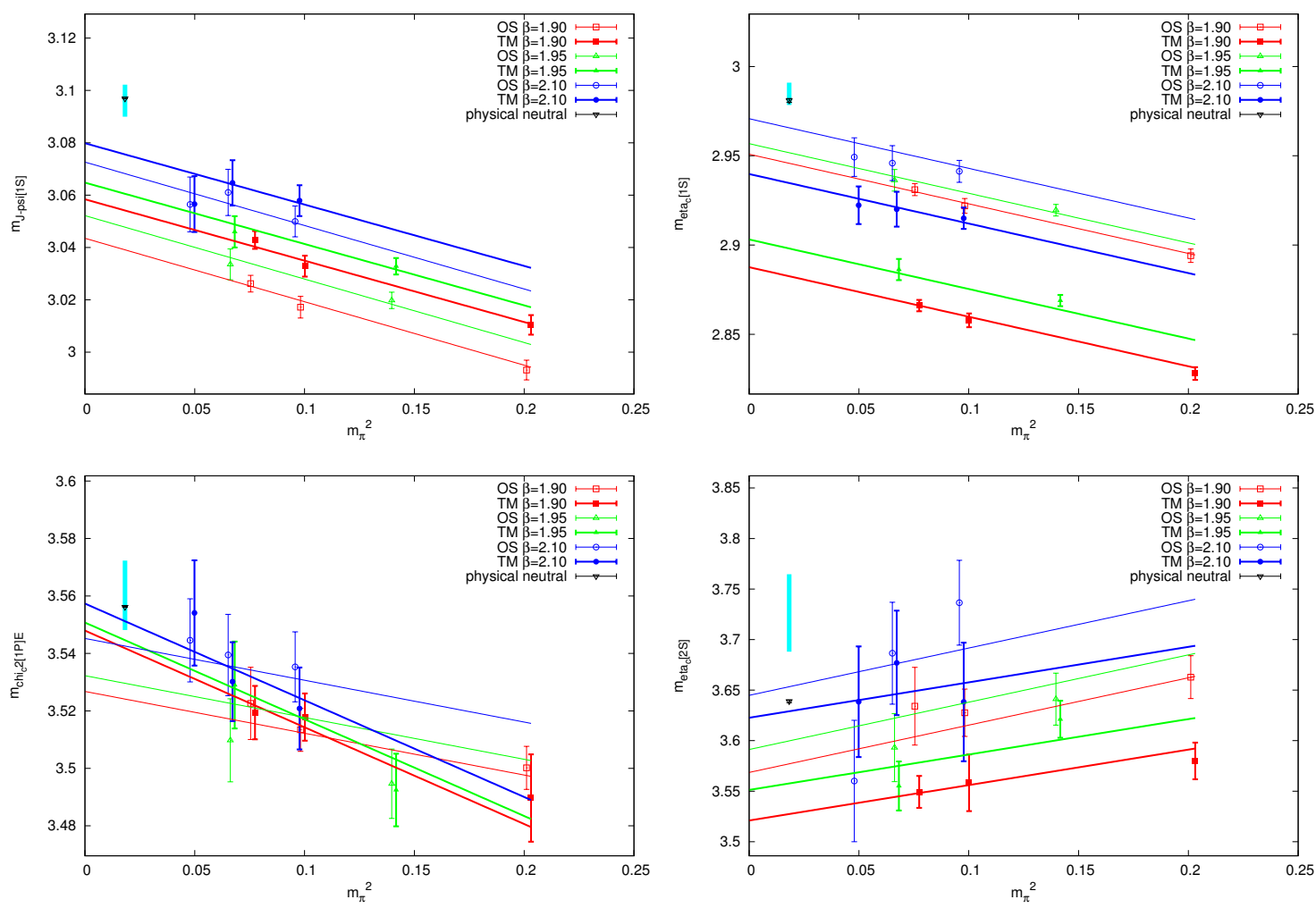

Figure 2: Combined chiral and continuum extrapolations in the charmonium sector: $J / \psi\left(J^{\mathscr{P} \mathscr{C}}=\right.$ $1^{--}$, top left $), \eta_{c}(1 S)\left(J^{\mathscr{P} \mathscr{C}}=0^{-+}\right.$, top right), $\chi_{c 2}(1 P)\left(J^{\mathscr{P} \mathscr{C}}=2^{++}\right.$, bottom left $), \eta_{c}(2 S)\left(J^{\mathscr{P} \mathscr{C}}=\right.$ $0^{-+}$, bottom right). PDG values of the masses [28] vs. results of our extrapolations: $3096.920(10)$ $\mathrm{MeV}$ vs. 3096(6) $\mathrm{MeV}\left(\chi^{2} /\right.$ d.o.f. of our fit: 0.36), 2981.1(1.1) $\mathrm{MeV}$ vs. $2985(6) \mathrm{MeV}\left(\chi^{2} /\right.$ d.o.f. $=$ $0.54), 3556.20(9) \mathrm{MeV}$ vs. $3560(12) \mathrm{MeV}\left(\chi^{2} /\right.$ d.o.f. $\left.=0.53\right)$, 3638.9(1.3) $\mathrm{MeV}$ vs. $3726(38) \mathrm{MeV}$ $\left(\chi^{2} /\right.$ d.o.f. $\left.=0.85\right)$, respectively.
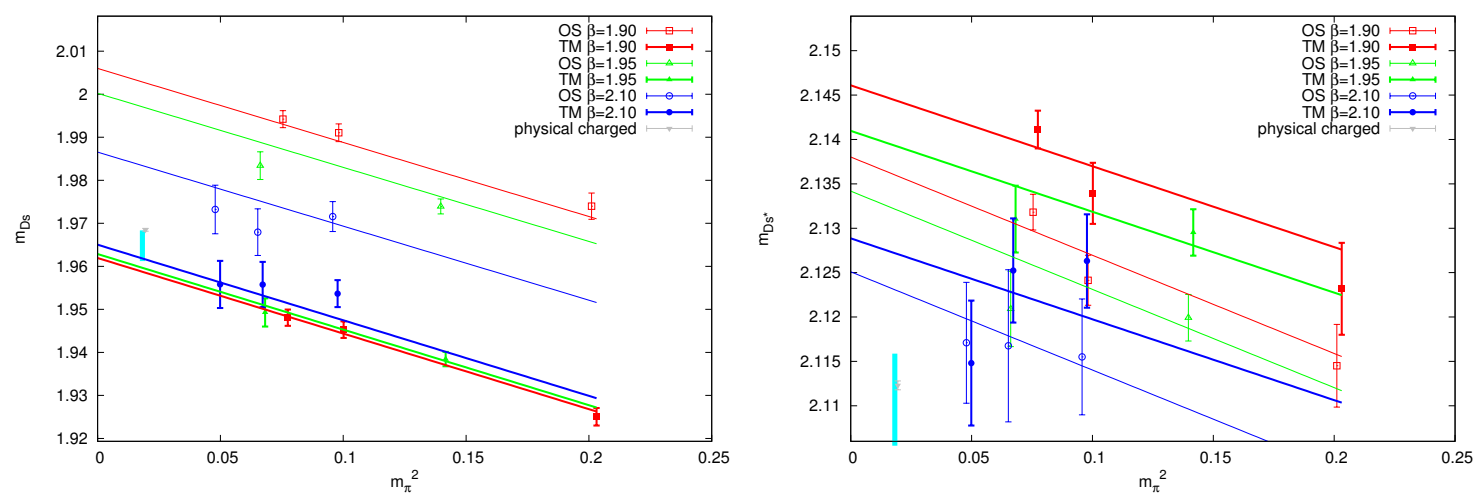

Figure 3: Combined chiral and continuum extrapolations in the $D_{s}$ sector: $D_{s}\left(J^{\mathscr{P}}=0^{-}\right.$, left $), D_{s}^{*}$ $\left(J^{\mathscr{P}}=1^{-}\right.$, right). PDG values of the masses [28] vs. results of our extrapolations: 1968.49 (32) $\mathrm{MeV}$ vs. $1964.8(3.6) \mathrm{MeV}\left(\chi^{2} /\right.$ d.o.f. $\left.=1.24\right), 2112.3(5) \mathrm{MeV}$ vs. $2110.7(5.2) \mathrm{MeV}\left(\chi^{2} /\right.$ d.o.f. $=$ 1.08), respectively. 

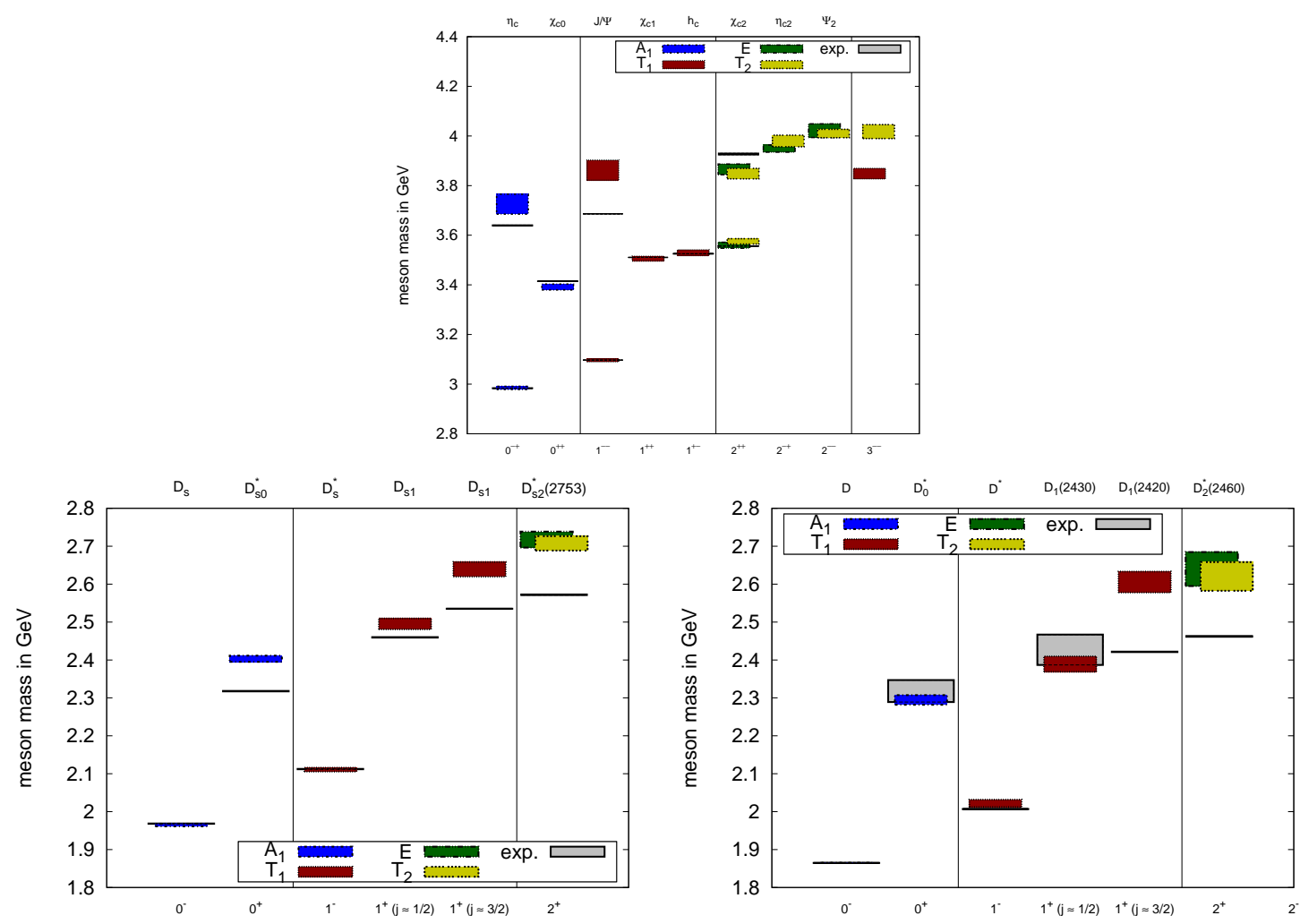

Figure 4: Spectrum plots for charmonium (top), $D_{s}$ mesons (bottom left) and $D$ mesons (bottom right). The black lines/grey boxes correspond to the PDG values [28] (if available; the line/box widths correspond to the experimental uncertainties and/or resonance widths), while the coloured boxes are our lattice results in different representations of the cubic group (extrapolated to the continuum limit and the physical quark masses).

our fitting ansätze give good description of lattice data and agreement with PDG values [28] for the ground states of given channels $\left(J / \psi, \eta_{c}(1 S), \chi_{c 2}(1 P), D_{s}, D_{s}^{*}\right)$ at the per-mille level. One of the presented cases $\left(\eta_{c}(2 S)\right)$ is an excited state (in the $J^{\mathscr{P} \mathscr{C}}=0^{-+}$channel) and we observe discrepancy with respect to the PDG value. We plan to investigate the sources of such discrepancies further - in the cases of excited states, they are most probably due to short plateaus and hence a systematic analysis of uncertainties from the choice of the plateau fitting range is needed.

Finally, we present the summary of our results in Fig. 4, where we compare our latticeextracted meson masses for charmonium, $D_{s}$ and $D$ mesons with PDG values [28]. Our results at this stage are still preliminary - in the near future we will extend our analysis [29] by including the missing quark mass at one of the lattice spacings and by investigating in more detail the sources of systematic uncertainties, such as the ones related to the choice of the plateau range (particularly for excited states), finite volume effects and the choice of the fitting ansatz to extrapolate to the physical pion mass and the continuum limit. Nevertheless, we already have rather good control over quark mass effects and discretization effects. For many cases, we obtain good agreement with PDG values, especially for ground states, where the plateau quality is good. Note that in certain 
cases we don't expect agreement with experiment, since we assume a dominating $q \bar{q}$ structure (whereas e.g. the $D_{s 0}^{*}$ might be a tetraquark) and that the particles are stable (while e.g. the $D_{0}^{*}$ can decay to $D+\pi$ and hence should possibly be treated with more advanced lattice techniques).

\section{Acknowledgments}

We acknowledge support by the Emmy Noether Programme of the DFG (German Research Foundation), grant WA 3000/1-1. This work was supported in part by the Helmholtz International Center for FAIR within the framework of the LOEWE program launched by the State of Hesse. Calculations on the LOEWE-CSC and on the on the FUCHS-CSC high-performance computer of the Frankfurt University were conducted for this research. We would like to thank HPC-Hessen, funded by the State Ministry of Higher Education, Research and the Arts, for programming advice.

\section{References}

[1] S. J. Dong et al., PoS LATTICE 2009, 090 (2009) [arXiv:0911.0868 [hep-ph]].

[2] T. Burch et al., Phys. Rev. D 81, 034508 (2010) [arXiv:0912.2701 [hep-lat]].

[3] J. J. Dudek et al., Phys. Rev. D 82, 034508 (2010) [arXiv:1004.4930 [hep-ph]].

[4] D. Mohler and R. M. Woloshyn, Phys. Rev. D 84, 054505 (2011) [arXiv:1 103.5506 [hep-lat]].

[5] Y. Namekawa et al. [PACS-CS Collaboration], Phys. Rev. D 84, 074505 (2011) [arXiv:1104.4600 [hep-lat]].

[6] G. S. Bali et al., PoS LATTICE 2011, 135 (2011) [arXiv:1108.6147 [hep-lat]].

[7] G. S. Bali, S. Collins and C. Ehmann, Phys. Rev. D 84, 094506 (2011) [arXiv:1110.2381 [hep-lat]].

[8] L. Liu et al. [Hadron Spectrum Collaboration], JHEP 1207, 126 (2012) [arXiv:1204.5425 [hep-ph]].

[9] Y. B. Yang et al. [CLQCD Collaboration], Phys. Rev. D 87, 014501 (2013) [arXiv:1206.2086 [hep-lat]].

[10] R. J. Dowdall et al., Phys. Rev. D 86, 094510 (2012) [arXiv:1207.5149 [hep-lat]].

[11] G. Moir et al., JHEP 1305, 021 (2013) [arXiv:1301.7670 [hep-ph]].

[12] B. A. Galloway et al. [HPQCD Collaboration], PoS LATTICE 2014, 092 (2014) [arXiv:1411.1318 [hep-lat]].

[13] P. Pérez-Rubio, S. Collins and G. S. Bali, Phys. Rev. D 92, 034504 (2015) [arXiv:1503.08440 [hep-lat]].

[14] M. Gong et al., PoS Lattice 2010, 106 (2010) [arXiv:1103.0589 [hep-lat]].

[15] D. Mohler, S. Prelovsek and R. M. Woloshyn, Phys. Rev. D 87, 034501 (2013) [arXiv:1208.4059 [hep-lat]].

[16] L. Liu et al., Phys. Rev. D 87, 014508 (2013) [arXiv:1208.4535 [hep-lat]].

[17] S. Prelovsek and L. Leskovec, Phys. Rev. Lett. 111, 192001 (2013) [arXiv:1307.5172 [hep-lat]].

[18] Y. Ikeda et al. [HAL QCD Collaboration], Phys. Lett. B 729, 85 (2014) [arXiv:1311.6214 [hep-lat]].

[19] C. B. Lang et al., Phys. Rev. D 90, 034510 (2014) [arXiv:1403.8103 [hep-lat]].

[20] S. Prelovsek et al., Phys. Rev. D 91, 014504 (2015) [arXiv:1405.7623 [hep-lat]].

[21] A. L. Guerrieri et al., PoS LATTICE 2014, 106 (2014) [arXiv:1411.2247 [hep-lat]].

[22] M. Kalinowski and M. Wagner, arXiv:1509.02396 [hep-lat], accepted in Phys. Rev. D.

[23] R. Baron et al., JHEP 1006, 111 (2010) [arXiv:1004.5284 [hep-lat]].

[24] Y. Iwasaki, K. Kanaya, T. Kaneko and T. Yoshie, Phys. Rev. D 56 (1997) 151 [hep-lat/9610023].

[25] R. Frezzotti and G. C. Rossi, JHEP 0408, 007 (2004) [arXiv: hep-lat/0306014].

[26] R. Frezzotti and G. C. Rossi, Nucl. Phys. Proc. Suppl. 128, 193 (2004) [arXiv:hep-lat/0311008].

[27] R. Frezzotti and G. C. Rossi, JHEP 0410, 070 (2004) [arXiv:hep-lat/0407002].

[28] K.A. Olive et al. (Particle Data Group), Chinese Physics C38, 090001 (2014).

[29] K. Cichy, M. Kalinowski and M. Wagner, in preparation. 